\title{
Diagnosis of Nontuberculous Mycobacterial Infections
}

\author{
Jakko van Ingen, $\mathrm{MD}, \mathrm{PhD}^{1}$ \\ ${ }^{1}$ Department of Medical Microbiology, Radboud University Nijmegen \\ Medical Center, Nijmegen, The Netherlands \\ Semin Respir Crit Care Med 2013;34:103-109.
}

\begin{abstract}
Address for correspondence Jakko van Ingen, MD, PhD, Department of Medical Microbiology, Radboud University Nijmegen Medical Center, P.O. Box 9101, 6500 HB Nijmegen, The Netherlands (e-mail: vaningen.jakko@gmail.com).
\end{abstract}

\begin{abstract}
Keywords

- nontuberculous mycobacteria

- diagnosis

- laboratory techniques

The nontuberculous mycobacteria (NTM) are typically environmental organisms residing in soil and water. Although generally of low pathogenicity to humans, NTM can cause a wide array of clinical diseases; pulmonary disease is most frequent, followed by lymphadenitis in children, skin disease by M. marinum (particularly in fish tank fanciers), and other extrapulmonary or disseminated infections in severely immunocompromised patients. Of the $>140$ NTM species reported in the literature, 25 species have been strongly associated with NTM diseases; the remainder are environmental organisms rarely encountered in clinical samples. Correct species identification is very important because NTM species differ in their clinical relevance. Further, NTM differ strongly in their growth rate, temperature tolerance, and drug susceptibility. The diagnosis of NTM disease is complex and requires good communication between clinicians, radiologists, and microbiologists. Isolation of $M$. kansasii and (in northwestern Europe) M. malmoense from pulmonary specimens usually indicates disease, whereas Mycobacterium gordonae and, to a lesser extent, $M$. simiae or $M$. chelonae are typically contaminants rather than causative agents of true disease. Mycobacterium avium complex (MAC), M. xenopi, and M. abscessus form an intermediate category between these two extremes. This review covers the clinical and laboratory diagnosis of NTM diseases and particularities for the different disease types and patient populations. Because of limited sensitivity and specificity of symptoms, radiology, and direct microscopy of clinical samples, culture remains the gold standard. Yet culture is time consuming and demands the use of multiple media types and incubation temperatures to optimize the yield. Outside of reference centers, such elaborate culture algorithms are scarce.
\end{abstract}

\section{Background}

The nontuberculous mycobacteria (NTM) are a grouping of all Mycobacterium species other than the obligate pathogens $M$. tuberculosis complex and M. leprae. They are typically environmental organisms residing in soil and natural as well as treated water. ${ }^{1}$ Although generally of low pathogenicity to humans, NTM can cause a wide array of clinical diseases; pulmonary disease is most frequent, followed by lymphadenitis in children, skin disease (by M. marinum, particularly in fish tank fanciers), and other extrapulmonary or disseminated infections in the severely immunocompromised. ${ }^{2}$ of the

Issue Theme Mycobacterial Infections: Guest Editor, Charles L. Daley, MD.
$>140$ NTM species now reported in the literature, some 25 species have been strongly associated with these NTM diseases; the remainder are true environmental organisms rarely encountered in clinical samples.

NTM differ strongly in their growth rate, temperature tolerance, and drug susceptibility. ${ }^{1-4}$ Owing to the differences in patient populations with their underlying lung diseases or immunodeficiencies as well as between the causative mycobacteria, diagnosis of NTM disease is complex and requires good communication between clinicians, radiologists, and microbiologists.
Copyright @ 2013 by Thieme Medical Publishers, Inc., 333 Seventh Avenue, New York, NY 10001, USA. Tel: +1(212) 584-4662. 


\section{Clinical Diagnosis of NTM Diseases}

\section{NTM Lung Disease}

Because NTM are environmental bacteria that humans encounter on a daily basis, ${ }^{1}$ diagnosing pulmonary NTM disease is not straightforward: a single positive culture from nonsterile sources including the respiratory or digestive tract need not indicate infection or disease. The American Thoracic Society (ATS) and Infectious Diseases Society of America (IDSA) have issued statements including a set of criteria to differentiate casual NTM isolation from true pulmonary NTM disease; these are summarized in - Table 1. ${ }^{5}$ In short, these criteria denote that to diagnose pulmonary NTM disease, clinical, radiological, and microbiological evidence of disease should be gathered.

Symptoms are generally nonspecific, in part owing to frequent underlying conditions. Most patients present with a chronic cough, with or without sputum production or hemoptysis, and slowly progressive fatigue or malaise. Constitutional symptoms (weight loss, fever, night sweats) are less frequent-occurring in 30 to $50 \%$ of patients-and often indicate advanced disease. ${ }^{5-7}$

Radiological abnormalities are more specific and generally follow two distinct patterns. The first manifestation is characterized radiologically by bronchiectasis and nodular lesions, mostly involving the lingula and middle lobe; the second is characterized by fibrocavitary lesions that mostly involve the upper lobes and resemble pulmonary tuberculosis. $^{5,8,9}$ Mixed types do occur, as do single large nodular lesions, mimicking malignancy. ${ }^{5}$ In cavitary disease, previous authors have suggested that NTM lung disease is characterized by thin-walled cavities, whereas tuberculosis would present with thick-walled cavities $^{8}$; a review of these and later studies has refuted the use of cavity appearance as a diagnostic tool. ${ }^{9}$ Cavitary lesions can occur in pulmonary malignancy and sarcoidosis as well as in infections by nonmycobacterial pathogens including fungi and Nocardia species. ${ }^{10}$ In nodular bronchiectatic disease, the combination of bronchiectasis, multiple small nodules, and a "tree-in-bud" pattern suggestive of bronchiolitis is quite specific for NTM lung disease. In a series of 105 patients with suggestive computed tomographic (CT) findings in South Korea, 34\% were diagnosed with NTM lung disease based on microbiological findings; the remainder were diagnosed with nonspecific bronchiolitis and bronchiectasis (50\%), diffuse panbronchiolitis (8\%), tuberculosis (6\%), or other diseases (2\%). ${ }^{11}$ Similar abnormalities can be seen in immunocompromised patients diagnosed with pulmonary nocardiosis. ${ }^{10}$ Hence other infectious (e.g., nocardiosis, fungal infection, tuberculosis) and noninfectious diseases (e.g., sarcoidosis) that can present with similar clinical and radiographic features have to be properly excluded before a firm diagnosis of NTM lung disease is made. Even in otherwise successful treatment, radiographic abnormalities may persist or even appear to increase in size; only small nodules tend to disappear during successful treatment. ${ }^{12}$

The third piece of evidence comes from the microbiology and pathology laboratories. To diagnose NTM lung disease

Table 1 Summary of the American Thoracic Society diagnostic criteria for pulmonary nontuberculous mycobacterial infection

\begin{tabular}{|l|}
\hline Clinical \\
\hline $\begin{array}{l}\text { 1. Pulmonary symptoms, nodular or cavitary opacities on chest radiograph, or ahigh-resolution computed tomographic } \\
\text { scan that shows multifocal bronchiectasis with multiple small nodules }\end{array}$ \\
\hline and \\
\hline 2. Appropriate exclusion of other diagnoses. \\
\hline Microbiologic \\
\hline $\begin{array}{l}\text { 1. Positive culture results from at least two separate expectorated sputum samples (If the results from the initial sputum } \\
\text { samples are nondiagnostic, consider repeat sputum acid-fast bacillus (AFB) smears and cultures) }\end{array}$ \\
\hline or \\
\hline 2. Positive culture results from at least one bronchial wash or lavage \\
\hline or \\
\hline $\begin{array}{l}\text { 3. Transbronchial or other lung biopsy with mycobacterial histopathological features (granulomatous inflammation } \\
\text { or AFB) and positive culture for NTM or biopsy showing mycobacterial histopathological features (granulomatous } \\
\text { inflammation or AFB) and one or more sputum or bronchial washings that are culture positive for NTM }\end{array}$ \\
\hline $\begin{array}{l}\text { 4. Expert consultation should be obtained when NTM are recovered that are either infrequently encountered } \\
\text { or that usually represent environmental contamination }\end{array}$ \\
\hline $\begin{array}{l}\text { 5. Patients who are suspected of having NTM lung disease but who do not meet the diagnostic criteria should be } \\
\text { followed until the diagnosis is firmly established or excluded }\end{array}$ \\
\hline $\begin{array}{l}\text { 6. Making the diagnosis of NTM lung disease does not, per se, necessitate the institution of therapy, which is a } \\
\text { decision based on potential risks and benefits of therapy for individual patients }\end{array}$ \\
\hline
\end{tabular}

Source: Adapted from Griffith DE, Aksamit T, Brown-Elliott BA, et al; ATS Mycobacterial Diseases Subcommittee; American Thoracic Society; Infectious Disease Society of America. An official ATS/IDSA statement: diagnosis, treatment, and prevention of nontuberculous mycobacterial diseases. Am J Respir Crit Care Med 2007;175(4):367-416. 
using the ATS diagnostic criteria, a set of at least three respiratory specimens should be obtained and sent for microbiological analysis whenever possible. Sampling intervals should be up to several weeks, although the exact timing has not been investigated. Short intervals between sampling pose the risk of interpreting accidental temporary presence of NTM in the airways after environmental exposure as a sign of disease. Of these three or more specimens, at least two should yield growth of the same NTM species for a solid diagnosis of NTM lung disease. Apart from the commonsense exclusion of occasional presence after environmental exposure or even of laboratory contamination, this requisite stems from one study which demonstrated that radiological evidence of disease (infiltrates or cavitary lesions) and progression was found in $98 \%$ of the patients who had two or more positive sputum cultures for M. avium complex, versus just $2 \%$ in those with a single positive culture during 12 months of observation. For $97 \%$ of patients, the first two positive cultures grew from the initial three sputum specimens. ${ }^{13}$

This microbiological criterion may be less applicable to the nodular-bronchiectatic type of NTM lung disease because these patients may produce less or no sputum. To diagnose nodular bronchiectatic NTM lung disease, bronchoalveolar lavage (BAL) fluid culture may be more sensitive than sputum culture. $^{14,15}$ Similarly, in a case series of 31 patients with mainly nodular bronchiectatic MAC disease, 45\% needed bronchoscopy or lung biopsy for diagnosis because sputum cultures were nondiagnostic. $^{7}$

Histological or cytological analysis of respiratory samples can be useful in difficult cases, including patients who do not produce sputum and will probably only produce a single positive culture from BAL, to ensure that the disease process is characterized by granulomatous inflammation. ${ }^{5}$ Especially in the immunocompromised, granuloma formation may be impaired, and the central caseous necrosis associated with tuberculosis may be absent. ${ }^{16}$

\section{Extrapulmonary and Disseminated Disease}

Lymphadenitis caused by NTM usually affects lymph nodes in a single site. The submandibular and cervical lymph nodes are most frequently affected, although axillary and inguinal lymphadenitis has been observed. Patients are typically (though not exclusively) children, under the age of 8 years. This age differs for the different species that cause lymphadenitis, but the background of this phenomenon remains elusive. ${ }^{17}$ This disease is relatively benign, and most patients present with an enlarged lymph node without constitutional symptoms; in more advanced disease, fluctuating masses with violaceous overlying skin are seen. Fine-needle aspirates or excised lymph nodes are the specimen of choice to obtain microbiological evidence of NTM disease. In fine-needle aspirates, molecular tools are likely more sensitive than culture, in part because these can detect the more fastidious $\operatorname{NTM}$ (e.g., M. haemophilum and “M. tilburgii"). ${ }^{18,19}$

The skin disease caused by $M$. marinum is characterized by single papulonodular, verrucose, or ulcerated granulomatous lesions, mostly on the hand or lower arm; single lesions may progress to form multiple lesions in a typical sporotrichoid pattern if left untreated. ${ }^{20}$ Taking a proper history is important to obtain evidence for contact with potential sources of M. marinum. This infection is most commonly seen in fish tank fanciers, though swimming pool visits, diving, and contact with fish have all been associated with development of $M$. marinum infection. ${ }^{20}$ Skin biopsies are the optimal specimens to obtain laboratory confirmation of infection but should be sent for histological examination as well as culture. ${ }^{20}$ The relative sensitivities and specificities of culture and histology have not been sufficiently studied.

Mycobacterium fortuitum can cause a similar skin disease, usually consisting of a single lesion, though these infections tend to occur after trauma, in surgical wounds, ${ }^{21}$ or in injection sites. ${ }^{2,5}$

Disseminated disease affects the immunocompromised, particularly patients with human immunodeficiency virus/ acquired immunodeficiency syndrome (HIV/AIDS), hematological malignancies, or those treated with immunosuppressive drugs after solid organ transplantation. ${ }^{2,5}$ More recently recognized risk groups are patients treated with the so-called biologicals(e.g., anti-tumor necrosis factor agents) for immune-mediated inflammatory diseases. ${ }^{22}$ Disseminated NTM diseases present as two distinct clinical syndromes. The first is a disease characterized by fever of unknown origin and generalized lymphadenopathy, although the latter may only develop over time or be most pronounced in abdominal lymph nodes. Owing to bone marrow infiltration, this disease may be accompanied by leucopenia or pancytopenia. ${ }^{5,23}$ This disease type is most strongly associated with MAC and $M$. simiae and has occurred in HIV/AIDS, hematological malignancies, and after solid organ transplantation. Diagnosis is usually by blood culture; staining and culture of biopsy specimens of bone marrow offer a similar (60 to $75 \%)^{23,24}$ or slightly higher sensitivity. ${ }^{25}$ Two studies have reported that staining and culture of liver biopsy specimens may provide a faster and slightly more sensitive alternative. ${ }^{26,27}$

The second manifestation is a disseminated skin disease that presents with nodules, subcutaneous abscesses, pustules, ulcers, or combinations thereof. This disease manifestation has been associated with rapid growers (M. abscessus, M. chelonae) and M. haemophilum and tends to affect patients with hematological malignancies or solid organ transplants but not HIV/AIDS. ${ }^{2,5,28}$ The reason for its absence in HIV/AIDS patients is unknown, and the skin tropism has been attributed to the preference of the causative species for lower temperatures $\left(30^{\circ} \mathrm{C}\right.$ rather than $37^{\circ} \mathrm{C}$ ), although this concept has not been experimentally tested. This disease manifestation is usually diagnosed by histological examination of skin biopsy specimens and culture. Granulomas are found in 30 to $50 \%$ of cases, ${ }^{21,29}$ thus rendering culture most important in the diagnosis. The exact sensitivities and specificities of these approaches have not been systematically studied.

\section{Laboratory Diagnosis}

To make a firm diagnosis of NTM disease in an individual patient, culture of representative clinical specimens and histological examination of tissue biopsy specimens are 
generally necessary. Given the importance of culture in the diagnosis of NTM disease, it is important to realize for microbiologists and clinicians that good communication between both parties may actually help to optimize culture conditions according to the particularities of the patient and therewith increase the sensitivity of culture and laboratory diagnosis of NTM disease.

\section{Microbiology: Sample Decontamination and Smear Microscopy}

Smear microscopy is mostly done in a two-step procedure, where samples are screened by fluorochrome (auramine) staining and positives are confirmed by classical Ziehl-Neelsen staining. Fluorochrome staining is the screening method because of its high sensitivity but low specificity. NTM are as likely as $M$. tuberculosis to be detected by fluorochrome staining. 30

To lower the loads of commensal flora associated with the human airways and digestive tract and thus overgrowth of cultures, sputum and bronchoalveolar lavage fluid as well as feces samples are decontaminated prior to inoculating them on selective media for Mycobacterium culture. Several different protocols for respiratory sample decontamination have been tried. Decontamination by $1 \%$ n-acetyl-L-cysteine ( NaLC)-NaOH is most commonly used; An increase of NaLC$\mathrm{NaOH}$ concentrations from $1 \%$ to $1.25 \%$ lowers contamination rates but also leads to a $10 \%$ decrease in detection of mycobacteria in culture and is not recommended. ${ }^{31}$ Sulfuric acid (final concentration 3\%) was recently shown to improve detection rates of NTM specifically (no influence on $M$. tuberculosis), compared with $1 \%$ NaLC-NaOH, by liquid culture. $^{32}$

For samples frompatients with cystic fibrosis (CF), which differ in their chemical composition as well their commensal flora, different approaches have been tested. In sputum samples of CF patients, decontamination by $0.25 \% / 1 \%$ NALC$\mathrm{NaOH}$, followed by $5 \%$ oxalic acid treatment, reduced the contamination rate from $74 \%$ of Lowenstein-Jensen slants or $36 \%$ of BacTec vials (BDBiosciences, Sparks, MD) (for NaLC$\mathrm{NaOH}$ alone) to only $5 \%$ and $3 \%^{33}$; yet, in a multisite reproducibility study, this method performed well only in acid-fast bacillus (AFB) smear positive samples. ${ }^{34}$ In a study comparing $1 \%$ chlorhexidine alone to $0.25 \% / 1 \%$ NaLC-NaOH followed by $5 \%$ oxalic acid in 827 sputum samples of CF patients, the former yielded twice as many NTM-positive cultures (6.50 vs $3.25 \%$ ), despite a higher contamination rate after chlorhexidine treatment (20 vs 14.2\%). ${ }^{35}$ Hence, it is important for mycobacteriology laboratories to know if a specimen comes from a CF patient or not-another example of a setting in which good communication can significantly improve clinical diagnostics.

\section{Culture Conditions-Media}

The choice of media for primary isolation largely determines the sensitivity. Liquid media are, in general, more sensitive than solid media such as Lowenstein-Jensen, Ogawa, Coletsos, and Middlebrook 7H10/7H11. ${ }^{36,37}$ Several studies have revealed that the widely used automated nonradiometric Mycobacteria Growth Indicator Tube (MGIT) method and its predecessor the radiometric BacTec460 method (both: BD Biosciences, Sparks, MD), both applying liquid media, were of about equal sensitivity; the slightly higher sensitivity of BacTec460(BD) observed in some studies was largely outweighed by its labor-intensive handling and use of radioactive materials with inherent safety concerns. ${ }^{38,39}$ Both these liquid culture systems apply an antibiotic supplement to suppress overgrowth of other bacteria and fungi, labeled PANTA, the acronym for polymyxin B (50 U/mL), amphotericin B $(5 \mu \mathrm{g} / \mathrm{mL})$, nalidixic acid $(20 \mu \mathrm{g} / \mathrm{mL})$, trimethoprim $(5 \mu \mathrm{g} / \mathrm{mL})$, and azlocillin $(10 \mu \mathrm{g} / \mathrm{mL})$.

There are more automated nonradiometric liquid culture systems available, including the MB BacT (bioMérieux, Durham, NC) and the recently renewed VersaTREK system (formerly known as ESP culture system II; Trek Diagnostics, Cleveland, $\mathrm{OH}$ ). The MB BacT system (bioMérieux) has been shown to be as sensitive as the MGIT (BD Biosciences) method. ${ }^{37,40}$ The time to detection and percentage contaminated cultures were lower in MGIT (BD Biosciences). ${ }^{37,40}$ For the VersaTREK system (Trek Diagnostics), no comparative studies of primary culture are currently available.

\section{Culture Conditions-Temperature}

The comparative studies outlined here have offered important insights in the performance of the various culture methods. Nonetheless, they may not reveal the full sensitivity that can be obtained in the routine clinical setting because both the solid and the liquid media were generally incubated at a single temperature, $35^{\circ} \mathrm{C}$. This temperature optimum is relevant to $M$. tuberculosis, but it limits the recovery of NTM, many of which have a growth optimum at $30^{\circ} \mathrm{C}$, $^{1,3,5}$ Thus optimal recovery of NTM is likely to be reached if both solid and liquid media are used and incubated at both 35 and $30^{\circ} \mathrm{C}$. Whether a liquid and solid medium should be incubated at both temperatures, or a hybrid of liquid at $35^{\circ} \mathrm{C}$ and solid at $30^{\circ} \mathrm{C}$, or liquid media only but at both $30^{\circ} \mathrm{C}$ and $35^{\circ} \mathrm{C}$, as well as cost-effectiveness of these approaches have not yet been studied, despite the increasing clinical importance of NTM in many settings.

\section{Culture Conditions-Medium Enrichment}

As typically environmental organisms, the NTM are quite versatile in their metabolic needs. Yet, for a small number of species, enrichment of culture media is needed to allow growth of the organism. For human medicine, $M$. genavense and $M$. haemophilum are the most prominent examples. For $M$. haemophilum, an iron source (ferric ammonium citrate or hemin) has to be added to the medium, and the media are best incubated at $30^{\circ} \mathrm{C}^{28}$ For $M$. genavense, some success has been reported for media composed of blood, charcoal, caseine, and yeast extracts, acidified to $\mathrm{pH} 6.0 .^{41}$ Yet this species remains very difficult to culture. ${ }^{42}$ Perhaps the most intriguing example is M. tilburgii, which has not been successfully cultured to date, despite the presence of large numbers of bacteria in clinical samples. ${ }^{19}$ Local epidemiology can guide the use of supplemented media. 


\section{Species Identification}

Correct species identification is very important because NTM species differ in their clinical relevance. ${ }^{6,43}$ Isolation of M. kansasii and (in northwestern Europe) M. malmoense from pulmonary specimens indicates disease in $>70 \%$ of all patients. ${ }^{5,6,44}$ Mycobacterium gordonae and, to a lesser extent, $M$. simiae or $M$. chelonae, are typically contaminants rather than causative agents of true disease ${ }^{5,6}$ and MAC, $M$. xenopi, and $M$. abscessus form an intermediate category between these two extremes. $5,6,43$

The methods for identification of mycobacteria in clinical laboratories have changed dramatically over the past 2 decades. Molecular methods have now surpassed biochemical tests and high-performance liquid chromatography of cell wall mycolic acid content as the method of choice for NTM identification. ${ }^{45}$ Among molecular methods, two approaches are commonly used. The first are line probe assays, which are easy to perform, albeit costly, assays that allow a reasonable level of discrimination and will allow identification of the most frequently encountered species. Second is (partial) gene sequencing which allows a higher level of discrimination, often up to subspecies level, but is only feasible for laboratories with access to sequencing facilities. The target(s) selected for sequencing determine the discriminatory power: the hsp65 and rpoB genes and the 16S-23S internal transcribed spacer (ITS) offer high discriminatory power and can identify up to the subspecies level, ${ }^{46-48}$ whereas $16 \mathrm{~S}$ rRNA gene sequencing allows discrimination to the species level for most species, or at least to the complex level, particularly among the rapid growers ( $M$. fortuitum complex, M. chelonae-M. abscessus complex). ${ }^{4,48}$

A new tool for species identification of NTM is matrixassisted laser desorption ionization-time of flight (MALDITOF) mass spectrometry. ${ }^{49}$ The optimal method for protein extraction from mycobacteria and the exact discriminatory power of this method have not yet been established.

\section{Impact of Diagnostic Criteria}

Currently, both the British Thoracic Society (BTS) and the American Thoracic Society (ATS) have issued diagnostic criteria for nontuberculous mycobacterial (lung) disease. ${ }^{5,50}$ Both state that isolation of NTM from normally sterile body sites is proof of true NTM disease, after exclusion of sample or laboratory contamination. Histological evidence of granulomatous inflammation strengthens the diagnosis. Diagnostic criteria for pulmonary NTM disease are less straightforward. Both BTS and ATS criteria emphasize the importance of symptoms and radiographic features suggestive of mycobacterial disease but differ in the level of detail of their microbiological criteria. The BTS criteria state that "multiple isolates are needed from nonsterile sites to establish disease. ${ }^{50}$ The clinical presentation and any predisposing factors are also helpful." 50 The ATS criteria state that three separate respiratory specimens, produced over several days or weeks, should be analyzed and that two positive cultures with the same species are required for diagnosis. ${ }^{5}$ Only in the setting of nodular bronchiectatic disease with little or no sputum production, a single positive sputum from a BAL specimen may suffice to diagnose NTM lung disease. ${ }^{5}$ The previous 1997 ATS diagnostic criteria had less stringent radiological criteria but more stringent microbiological criteria requiring three positive cultures with the same species. Multiple studies have revealed that more patients meet the 2007 than the 1997 ATS diagnostic criteria, and this could lead to overdiagnosis and overtreatment of NTM lung disease in some. ${ }^{51,52}$ On the other hand, less strict criteria may decrease diagnostic delay. The sensitivity of the different diagnostic criteria has not been tested in clinical studies; this is particularly problematic in select patient populations, such as CF patients, whose underlying disease resembles NTM pulmonary disease in terms of both the symptoms and the radiological features.

\section{Perspective}

Despite tremendous advances in clinical and laboratory diagnosis of the different NTM diseases, diagnosing NTM diseases remains complicated. It is, of course, key to think of NTM as possible causative agents of disease. Then, because of limited sensitivity and specificity of symptoms, radiology, and direct microscopy of clinical samples, culture remains the gold standard. Yet culture is time consuming and demands the use of multiple media types and incubation temperatures to optimize the yield. Outside of reference centers, such elaborate culture algorithms are scarce.

Determining the clinical relevance of isolated NTM presents its own challenges. Three particular issues merit specific attention and should be subjects of future studies. First, it is now generally accepted that NTM species differ in their clinical relevance ${ }^{6}$; yet a single species may also differ in clinical relevance in different regions or settings, which adds another layer of complexity. ${ }^{53}$ To prevent unwarranted diagnoses and treatment of NTM disease as well as unnecessary diagnostic delay, it could be helpful to use separate, more stringent criteria for species of low, and less stringent criteria for species considered to be of high clinical relevance in the local setting. This stepped approach requires complete and up to date insight in locally prevalent NTM and their clinical relevance. In the absence of obligatory reporting for NTM, this too is an area where continuous contact between clinicians and microbiologists is important.

Virulence factors are a second understudied field in NTM. Yet detecting these may aid in determining the clinical relevance of isolated NTM. Here the species M. kansasii is the best example. In a previous case series, M. kansasii subtype 1 was most strongly associated with clinical disease, whereas subtypes 3 through 5 seemed nonpathogenic. ${ }^{54} \mathrm{~A}$ recent study of the ESX-1 virulence factor of $M$. tuberculosis also evaluated $M$. kansasii and found that $M$. kansasii subtype 1 had an active ESX-1 system, whereas this system was inactive in $M$. kansasii type $5 .{ }^{55}$ Assessing such virulence factors could be a valuable addition to species identification.

The third key issue in diagnosing NTM infections is the recognition of patients at increased risk for these diseases. Even though some predisposing conditions are very clear(e.g., HIV, immunosuppressive drug use) many others remain poorly understood. Even though preexistent lung diseases, 
including chronic obstructive pulmonary disease and $\mathrm{CF}$, are clear risk factors, it remains impossible to predict which individual patient will develop NTM disease, even though we are all exposed to NTM on a daily basis. ${ }^{56}$ This also should be an area of future studies.

\section{References}

1 Falkinham JO. Impact of human activities on the ecology of nontuberculous mycobacteria. Future Microbiol 2010;5(6):951-960

2 Wolinsky E. Nontuberculous mycobacteria and associated diseases. Am Rev Respir Dis 1979;119(1):107-159

3 Schulze-Röbbecke R, Buchholtz K. Heat susceptibility of aquatic mycobacteria. Appl Environ Microbiol 1992;58(6):1869-1873

4 van Ingen J, van der Laan T, Dekhuijzen R, Boeree MJ, van Soolingen D. In vitro drug susceptibility of 2275 clinical non-tuberculous Mycobacterium isolates of 49 species in the Netherlands. Int J Antimicrob Agents 2010;35(2):169-173

5 Griffith DE, Aksamit T, Brown-Elliott BA, et al; ATS Mycobacterial Diseases Subcommittee; American Thoracic Society; Infectious Disease Society of America. An official ATS/IDSA statement: diagnosis, treatment, and prevention of nontuberculous mycobacterial diseases. Am J Respir Crit Care Med 2007;175(4):367-416

6 van Ingen J, Bendien SA, de Lange WCM, et al. Clinical relevance of non-tuberculous mycobacteria isolated in the Nijmegen-Arnhem region, The Netherlands. Thorax 2009;64(6):502-506

7 Huang JH, Kao PN, Adi V, Ruoss SJ. Mycobacterium avium-intracellulare pulmonary infection in HIV-negative patients without preexisting lung disease: diagnostic and management limitations. Chest 1999;115(4):1033-1040

8 Albelda SM, Kern JA, Marinelli DL, Miller WT. Expanding spectrum of pulmonary disease caused by nontuberculous mycobacteria. Radiology 1985;157(2):289-296

9 Ellis SM. The spectrum of tuberculosis and non-tuberculous mycobacterial infection. Eur Radiol 2004;14(Suppl 3):E34-E42

10 Kanne JP, Yandow DR, Mohammed TL, Meyer CA. CT findings of pulmonary nocardiosis. AJR Am J Roentgenol 2011;197(2):W266-72

11 Koh WJ, Lee KS, Kwon OJ, Jeong YJ, Kwak SH, Kim TS. Bilateral bronchiectasis and bronchiolitis at thin-section CT: diagnostic implications in nontuberculous mycobacterial pulmonary infection. Radiology 2005;235(1):282-288

12 Fujiuchi S, Matsumoto H, Yamazaki Y, et al. Analysis of chest CT in patients with Mycobacterium avium complex pulmonary disease. Respiration 2003;70(1):76-81

13 Tsukamura M. Diagnosis of disease caused by Mycobacterium avium complex. Chest 1991;99(3):667-669

14 Sugihara E, Hirota N, Niizeki T, et al. Usefulness of bronchial lavage for the diagnosis of pulmonary disease caused by Mycobacterium avium-intracellulare complex (MAC) infection. J Infect Chemother 2003;9(4):328-332

15 Tanaka E, Amitani R, Niimi A, Suzuki K, Murayama T, Kuze F. Yield of computed tomography and bronchoscopy for the diagnosis of Mycobacterium avium complex pulmonary disease. Am J RespirCrit Care Med 1997;155(6):2041-2046

16 O'Connell ML, Birkenkamp KE, Kleiner DE, Folio LR, Holland SM, Olivier KN. Lung manifestations in an autopsy-based series of pulmonary or disseminated nontuberculous mycobacterial disease. Chest 2012;141(5):1203-1209

17 van Ingen J, van Soolingen D. Cervicofacial lymphadenitis caused by nontuberculous mycobacteria; host, environmental or bacterial factors? Int J Pediatr Otorhinolaryngol 2011;75:722-723

18 Bruijnesteijn Van Coppenraet ES, Lindeboom JA, Prins JM, Peeters MF, Claas EC, Kuijper EJ. Real-time PCR assay using fine-needle aspirates and tissue biopsy specimens for rapid diagnosis of mycobacterial lymphadenitis in children. J Clin Microbiol 2004;42(6):2644-2650
19 Hartwig NG, Warris A, van de Vosse E, et al. "Mycobacterium tilburgii" infection in two immunocompromised children: importance of molecular tools in culture-negative mycobacterial disease. J Clin Microbiol 2011;49(12):4409-4411

20 Petrini B. Mycobacterium marinum: ubiquitous agent of waterborne granulomatous skin infections. Eur J Clin Microbiol Infect Dis 2006;25(10):609-613

21 Uslan DZ, Kowalski TJ, Wengenack NL, Virk A, Wilson JW. Skin and soft tissue infections due to rapidly growing mycobacteria: comparison of clinical features, treatment, and susceptibility. Arch Dermatol 2006;142(10):1287-1292

22 van Ingen J, Boeree MJ, Dekhuijzen PNR, van Soolingen D. Mycobacterial disease in patients with rheumatic disease. Nat Clin Pract Rheumatol 2008;4(12):649-656

23 Kilby JM, Marques MB, Jaye DL, Tabereaux PB, Reddy VB, Waites $\mathrm{KB}$. The yield of bone marrow biopsy and culture compared with blood culture in the evaluation of HIV-infected patients for mycobacterial and fungal infections. Am J Med 1998;104(2): 123-128

24 Akpek G, Lee SM, Gagnon DR, Cooley TP, Wright DG. Bone marrow aspiration, biopsy, and culture in the evaluation of HIV-infected patients for invasive mycobacteria and histoplasma infections. Am J Hematol 2001;67(2):100-106

25 Ker CC, Hung CC, Huang SY, et al. Comparison of bone marrow studies with blood culture for etiological diagnosis of disseminated mycobacterial and fungal infection in patients with acquired immunodeficiency syndrome. J Microbiol Immunol Infect 2002; 35(2):89-93

26 Prego V, Glatt AE, Roy V, Thelmo W, Dincsoy H, Raufman JP. Comparative yield of blood culture for fungi and mycobacteria, liver biopsy, and bone marrow biopsy in the diagnosis of fever of undetermined origin in human immunodeficiency virus-infected patients. Arch Intern Med 1990;150(2):333-336

27 Cappell MS, Schwartz MS, Biempica L. Clinical utility of liver biopsy in patients with serum antibodies to the human immunodeficiency virus. Am J Med 1990;88(2):123-130

28 Saubolle MA, Kiehn TE, White MH, Rudinsky MF, Armstrong D. Mycobacterium haemophilum: microbiology and expanding clinical and geographic spectra of disease in humans. Clin Microbiol Rev 1996;9(4):435-447

29 Liao CH, Lai CC, Ding LW, et al. Skin and soft tissue infection caused by non-tuberculous mycobacteria. Int J Tuberc Lung Dis 2007; 11(1):96-102

30 Wright PW, Wallace RJ Jr, Wright NW, Brown BA, Griffith DE. Sensitivity of fluorochrome microscopy for detection of Mycobacterium tuberculosis versus nontuberculous mycobacteria. J Clin Microbiol 1998;36(4):1046-1049

31 Peres RL, Maciel EL, Morais CG, et al. Comparison of two concentrations of NALC-NaOH for decontamination of sputum for mycobacterial culture. Int J Tuberc Lung Dis 2009;13(12): 1572-1575

32 Buijtels PC, Petit PL. Comparison of $\mathrm{NaOH}-\mathrm{N}$-acetyl cysteine and sulfuric acid decontamination methods for recovery of mycobacteria from clinical specimens. J Microbiol Methods 2005;62(1): 83-88

33 Whittier S, Hopfer RL, Knowles MR, Gilligan PH. Improved recovery of mycobacteria from respiratory secretions of patients with cystic fibrosis. J Clin Microbiol 1993;31(4):861-864

34 Whittier S, Olivier K, Gilligan P, Knowles M, Della-Latta P; The Nontuberculous Mycobacteria in Cystic Fibrosis Study Group. Proficiency testing of clinical microbiology laboratories using modified decontamination procedures for detection of nontuberculous mycobacteria in sputum samples from cystic fibrosis patients. J Clin Microbiol 1997;35(10):2706-2708

35 Ferroni A, Vu-Thien H, Lanotte P, et al. Value of the chlorhexidine decontamination method for recovery of nontuberculous mycobacteria from sputum samples of patients with cystic fibrosis. J Clin Microbiol 2006;44(6):2237-2239 
36 Idigoras P, Beristain X, Iturzaeta A, Vicente D, Pérez-Trallero E. Comparison of the automated nonradiometricBactec MGIT 960 system with Löwenstein-Jensen, Coletsos, and Middlebrook 7H11 solid media for recovery of mycobacteria. Eur J Clin Microbiol Infect Dis 2000;19(5):350-354

37 Yan JJ, Huang AH, Tsai SH, Ko WC, Jin YT, Wu JJ. Comparison of the $\mathrm{MB} /$ BacT and BACTEC MGIT 960 system for recovery of mycobacteria from clinical specimens. Diagn Microbiol Infect Dis 2000; 37(1):25-30

38 Chew WK, Lasaitis RM, Schio FA, Gilbert GL. Clinical evaluation of the Mycobacteria Growth Indicator Tube (MGIT) compared with radiometric (Bactec) and solid media for isolation of Mycobacterium species. J Med Microbiol 1998;47(9):821-827

39 Tortoli E, Cichero P, Piersimoni C, Simonetti MT, Gesu G, Nista D. Use of BACTEC MGIT 960 for recovery of mycobacteria from clinical specimens: multicenter study. J Clin Microbiol 1999;37(11):3578-3582

40 Alcaide F, Benítez MA, Escribà JM, Martín R. Evaluation of the BACTEC MGIT 960 and the MB/BacT systems for recovery of mycobacteria from clinical specimens and for species identification by DNA AccuProbe. J Clin Microbiol 2000;38(1):398-401

41 Realini L, De Ridder K, Hirschel B, Portaels F. Blood and charcoal added to acidified agar media promote the growth of Mycobacterium genavense. Diagn Microbiol Infect Dis 1999;34(1):45-50

42 Thomsen VO, Dragsted UB, Bauer J, Fuursted K, Lundgren J. Disseminated infection with Mycobacterium genavense: a challenge to physicians and mycobacteriologists. J Clin Microbiol 1999;37(12):3901-3905

43 Koh WJ, Kwon OJ, Jeon K, et al. Clinical significance of nontuberculous mycobacteria isolated from respiratory specimens in Korea. Chest 2006;129(2):341-348

44 Hoefsloot W, van Ingen J, de Lange WCM, Dekhuijzen PNR, Boeree MJ, van Soolingen D. Clinical relevance of Mycobacterium malmoense isolation in The Netherlands. Eur Respir J 2009;34(4):926-931

45 Springer B, Stockman L, Teschner K, Roberts GD, Böttger EC. Twolaboratory collaborative study on identification of mycobacteria: molecular versus phenotypic methods. J Clin Microbiol 1996; 34(2):296-303

46 Roth A, Fischer M, Hamid ME, Michalke S, Ludwig W, Mauch H. Differentiation of phylogenetically related slowly growing mycobacteria based on 16S-23S rRNA gene internal transcribed spacer sequences. J Clin Microbiol 1998;36(1):139-147
47 McNabb A, Eisler D, Adie K, et al. Assessment of partial sequencing of the 65-kilodalton heat shock protein gene (hsp65) for routine identification of Mycobacterium species isolated from clinical sources. J Clin Microbiol 2004;42(7):3000-3011

48 Adékambi T, Colson P, Drancourt M. rpoB-based identification of nonpigmented and late-pigmenting rapidly growing mycobacteria. J Clin Microbiol 2003;41(12):5699-5708

49 Saleeb PG, Drake SK, Murray PR, Zelazny AM. Identification of mycobacteria in solid-culture media by matrix-assisted laser desorption ionization-time of flight mass spectrometry. J Clin Microbiol 2011;49(5):1790-1794

50 Subcommittee of the Joint Tuberculosis Committee of the British Thoracic Society. Management of opportunist mycobacterial infections: Joint Tuberculosis Committee Guidelines 1999. Thorax 2000;55(3):210-218

51 van Ingen J, Boeree MJ, de Lange WC, Dekhuijzen PN, van Soolingen D. Impact of new American Thoracic Society diagnostic criteria on management of nontuberculous mycobacterial infection. Am J RespirCrit Care Med 2007;176(4):418, author reply 419

52 Epson E, Cassidy M, Marshall-Olson A, Hedberg K, Winthrop KL. Patients with nontuberculous mycobacteria: comparison of updated and previous diagnostic criteria for lung disease. Diagn Microbiol Infect Dis 2012;74(1):98-100

53 Martín-Casabona N, Bahrmand AR, Bennedsen J, et al; Spanish Group for Non-Tuberculosis Mycobacteria. Non-tuberculous mycobacteria: patterns of isolation. A multi-country retrospective survey. Int J Tuberc Lung Dis 2004;8(10): 1186-1193

54 Taillard C, Greub G, Weber R, et al. Clinical implications of Mycobacterium kansasii species heterogeneity: Swiss National Survey. J Clin Microbiol 2003;41(3):1240-1244

55 Houben D, Demangel C, van Ingen J, et al. ESX-1-mediated translocation to the cytosol controls virulence of mycobacteria. Cell Microbiol 2012;14(8):1287-1298

56 Verregghen M, Heijerman HG, Reijers M, van Ingen J, van der Ent CK. Risk factors for Mycobacterium abscessus infection in cystic fibrosis patients; a case-control study. J Cyst Fibros 2012;11(4): 340-343 